\title{
The Design of Pneumatic System in High-Speed Bagging Machine
}

\author{
Fei ZHONG ${ }^{a}$, Qian-Cheng WAN ${ }^{b}$, Jia-Yi SONG ${ }^{c}$, Kai-Long LUd
}

Hubei University of Technology, No.28, Nanli Road, Hong-shan District, Wuchang, Wuhan, Hubei

Province, P. R. China 430068

qianrocket@hotmail.com

Keywords: Bagging machine, Pneumatic and vacuum system, Cylinder.

\begin{abstract}
The composite packaging bag is widespread used in packaging industry. In the past, the production of it is mostly done by manual, so manufacturer want to use automated machinery to improve efficiency and reduce intensity of labor. The pneumatic and vacuum system as a important part of this product need to be stably and reliably. In this article we use pneumatic system theory solve the problem of design and selection of this system. We calculated the diameter of the air pipe, the bore, stroke, air consumption of the cylinder, and torque of the rotary cylinders in a row. By this we selected the electromagnetic valve, air cylinder, and vacuum chucks. After design and product other parts, we have successfully tested the entire system and it meet the needs of automation and efficient. The product fill the gaps of the bagging machine. and it have been good use in the cooperative company's production process.
\end{abstract}

\section{Introduction}

Pneumatic system includes pneumatic transmission and control, which based on compressed air as a medium, use the pneumatic components to complete into the appropriate action, is an important means to achieve production automation[1]. In recent years, with the progress and development of science and technology, pneumatic technology matures have wide range of applications in the chemical industry, weaving spinning, medicine, electronics, bio-engineering, food, etc. domain, especially in a variety of automated production equipment and production lines.

Pneumatic technology obviously have a very clear advantage compared with other control and drive technology in the following aspects[2-4].Working medium is inexhaustible air, it cost low and no pollution. Control device have long life and high stablity. Gas deformation degree is big and make the pneumatic system buffering ability is strong. The system is lexible, adaptable, suitable for high humidity, electromagnetic radiation.

However, the pneumatic technology relative to other control and drive technology has the following problems. It has the huge noise, needs to add the silencer, difficult to maintain stability, not suitable for the signal demanding system. Compared with the hydraulic system, the pneumatic system output and stability is poor.

Although the pneumatic technology have some problems, it was widely used in packing machine with its obviously advantages. We also use the technology to realize automatization of our equipment.

\section{Project Design}

\section{Major Require and Technical Index}

The main technical parameters of the bagging machine; Bag specifications: inner membrane bag length $1080 \mathrm{~mm}$, width $580 \mathrm{~mm}$; paper bag length $830 \mathrm{~mm}$, width $460-550 \mathrm{~mm}$;production speed 20 30 pieces / $\mathrm{min}$; inner bag crawl length: fixed length $\pm 10 \mathrm{~mm}$;Outer bag delivery length: fixed length $\pm 15 \mathrm{~mm}$;inside and outside the bag length error: $\pm 3 \mathrm{~mm}$; bag machine hot knife temperature: $220^{\circ} \mathrm{C}$, error $\pm 3^{\circ} \mathrm{C}$. Functional requirement: power on self-test, automatic operation, manual operation, automatic reset.

\section{Major Designing Scheme}

According to the requirement combine with the pneumatic technology. We protocol the major scheme. 
In this system, the use of air compressors and vacuum pumps to provide positive and negative airdriven cylinder and vacuum suction cup to complete the corresponding action. Meanwhile we use various pneumatic valve to achieve output force, speed and direction of control. At the same time the use of cylinder magnetic sensors and vacuum pressure switch to detect action and pressure, transmission of signals to the PLC, to achieve feedback control. This picture shows the main scheme.

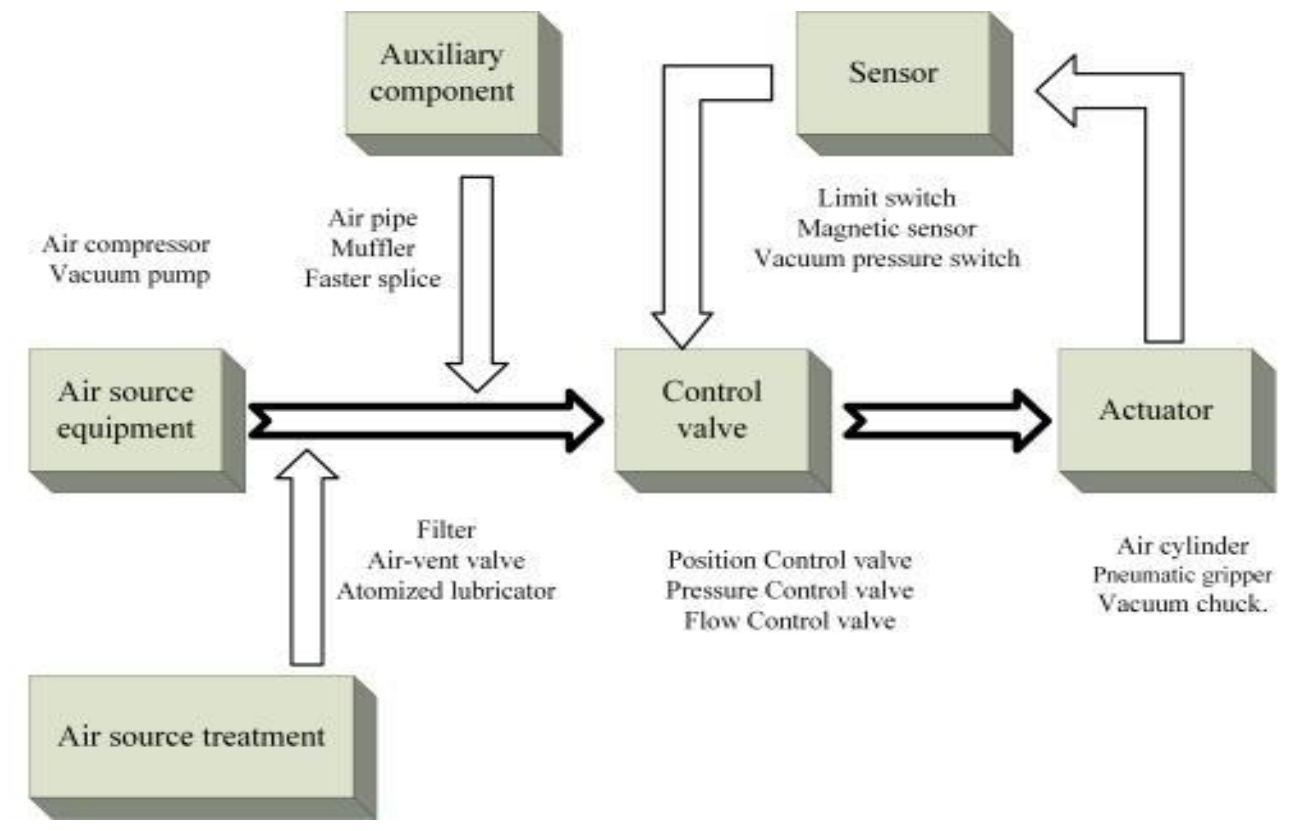

Figure 1 .The main designing scheme

\section{Theoretical Analysis and Calculation of Pneumatic System}

In the pneumatic system design, according to the actual requirements of a reasonable choice of pneumatic components. Selection of pneumatic components to consider the choice of flow, speed and output force requirements, taking into account the cost of pneumatic components and installation of rationality. Using pneumatic technology theory, calculate the basic parameters of pneumatic components, select a reasonable pneumatic components model.

\section{Air Supply, Trachea and Pneumatic Triple Pieces}

The system uses air compressors to provide compressed air, the use of vacuum pumps for the pneumatic system to provide negative pressure. We use the common pneumatic triple pieces as air handling equipment including air filters, pressure regulators and oil mist [4].

General pneumatic actuators required air pressure of $0.6-1.3 \mathrm{MPa}$. Taking into account the gas pressure in the gas pipeline in the consumption, the general gas source initial pressure is higher than the maximum pressure in the gas line about $20 \%$ to meet the pressure demand. If a low pressure is required in a part of the system, a relief valve can be used to supply the gas in the branch gas line. Considering the actual demand, the system uses the rated pressure of $0.6 \mathrm{MPa}$ air compressor, and select rated pressure $-600 \mathrm{MPa}$ rotary vane vacuum pump.

The diameter of the trachea directly affects the speed and stability of the pneumatic system, the trachea diameter calculation formula is as follows:

$$
s=\frac{A}{\sqrt{\eta C_{p}+1}}
$$

$\mathrm{S}$ : effective area of cylinder pipe $\quad\left(\mathrm{mm}^{2}\right)$

A: air pipe diameter $A=\frac{\pi}{4} d^{2} \quad\left(\mathrm{~mm}^{2}\right)$

$\eta$ :friction coefficient of air pipe 
steel tuben $=15$,

plastic pipen $=10$;

$\mathrm{C}_{\mathrm{p}}$ : air pipe Length and diameter ratio。

Pneumatic triple pieces through the flow calculation formula is as follows:

$$
Q=\frac{\pi\left(D^{2} v_{\max }+d^{2} l\right)(p+1.013) \times 60}{4 \times 1.013 \times 10^{6}}(L / \mathrm{min})
$$

$D$ : cylinder bore $\quad(\mathrm{mm})$

$V_{\max }:$ maximum cylinder speed $(\mathrm{mm} / \mathrm{s})$

$l$ : pipe length

( $m m)$

$P$ : working pressure $\quad\left(M P_{a}\right)$

$d$ :diameter of air pipe $\quad(\mathrm{mm})$

\section{Calculation of Cylinder Bore}

Cylinder bore diameter refers to the cylinder piston diameter, from the general cylinder theoretical output force formula can be derived Bore calculation formula is as follows:

When the cylinder thrust (piston rod out):

$$
D=\sqrt{\frac{4 F_{1}}{\pi P}}
$$

When the cylinder tension (piston rod out):

$$
D=\sqrt{\frac{4 F_{2}}{\pi P}+d^{2}}
$$

$D$ :cylinder bore $\quad(\mathrm{mm})$

$F_{1:}$ theoretical output thrust

$F_{2}$ :theoretical output tension

$P$ : work pressure $\left(M P_{a}\right)$

$d$ :Cylinder piston rod diameter $\quad(\mathrm{mm})$

But the actual selection of the actual output force will be greater than the theoretical output force to ensure the accuracy of the cylinder action. The actual output force is calculated as follows:

$$
F=\beta F_{0}
$$

$\beta$ is the cylinder load rate, the value determined by the load and speed of movement:

Table 1. Setting Word's margins.

\begin{tabular}{c|c|c|c}
\hline \multirow{2}{*}{ Static load } & \multicolumn{3}{|c}{ Inertial load $\boldsymbol{V ~ m m} / \mathbf{s}$} \\
\cline { 2 - 4 } & $\leq 100$ & $100 \sim 500$ & $\geq 500$ \\
\hline$\beta \leq 0.8$ & $\beta \leq 0.65$ & $\beta \leq 0.5$ & $\beta \leq 0.3$ \\
\hline
\end{tabular}

\section{Selection of Cylinder Stroke}

Cylinder stroke and its installation and the actual use of the situation, the cylinder stability action to meet the following conditions:

$$
F \leq \frac{F_{K}}{n_{k}}
$$

$F$ : maximum axial force

$F_{k}$ : longitudinal bending force

$n_{k}$ : stability factor 
generally take 1.5 - 40

When the ratio between the cylinder mounting length $\mathrm{L}$ and the bore diameter $\mathrm{d}$ is greater than 42 . 5:

$$
F_{k}=\frac{m \pi^{3} E d^{4}}{64 L^{2}}
$$

When the ratio of the cylinder installation length $L$ to the bore diameter $d$ is less than 42.5 :

$$
F_{k}=\frac{m \sigma_{y} \pi d^{4}}{4 m d^{2}+16 \alpha L^{2}}
$$

$m$ : bearing condition end factor

$\sigma:$ compressive strength of steel

$$
\sigma=3.9 \times 10^{8} P_{a}
$$

E:Elastic modulus of

$$
\text { steel } E=2.1 \times 10^{8} P_{a}
$$

$\alpha$ :Coefficient of steel $\alpha=1 / 5000$

\section{Calculation of Air Consumption of Cylinder}

Cylinder air consumption is the amount of compressed air consumed by the cylinder in the telescopic movement, is to determine the important indicators of pneumatic circuits

Theoretical conditions, the maximum cylinder air consumption is calculated as follows:

$$
q_{v \max }=\frac{470 D^{2} L(P+0.1) P}{t}
$$

qvmax:maximum air consumption $\quad(L / m i n)$

$D$ :cylinder bore $\quad(\mathrm{mm})$

L:cylinder stroke $\quad(\mathrm{mm})$

$P$ :working pressure $\quad\left(M P_{a}\right)$

$t$ :the time required for the reciprocating stroke of the piston(s)

\section{Torque Calculation for Rotary Cylinders}

An important execution cylinder in the present pneumatic system is a rotary cylinder for holding the conveyance of inner and outer bags. So rotating the cylinder torque is a very important technical parameters. Theoretical conditions, the blade-type rotary cylinder output torque formula is:

$$
M=A \cdot\left(D^{2}-d^{2}\right) b \cdot P \times 10^{-3}
$$

$\begin{array}{lc}M: \text { theoretical output torque } & \left(N^{*} \mathrm{~m}\right) \\ D: \text { outer diameter of rotor } & (\mathrm{mm}) \\ d: \text { internal diameter of rotor } & (\mathrm{mm}) \\ b: \text { blade width } & (\mathrm{mm}) \\ P \text { : working pressure } & \left(M P_{a}\right)\end{array}$

When the blade is a single leaf is to take $A=1 / 8$, for the double leaves take $A=1 / 4$.

Theoretical formula, the gear-rack rotary cylinder output torque formula:

$$
M=\frac{\pi}{8} \cdot D^{2} \cdot d_{f} \cdot p \times 10^{-3}(N \bullet m)
$$

$D$ :cylinder bore

$d_{f: \text { :gear index circle diameter }}$

$P$ :working pressure $(\mathrm{mm})$

$(\mathrm{mm})$

$\left(M P_{a}\right)$

Calculation of Vacuum Chucks 
In order to ensure that the vacuum sucker can safely complete the task, we must ensure that the suction force of the vacuum sucker is greater than the load capacity. In a certain negative pressure outside the case, the suction force of a single vacuum suction cup is determined by the actual area of the suction cup. The suction cup diameter D is selected as follows

$$
D \leq \sqrt{\frac{4 W t}{\pi P}}
$$

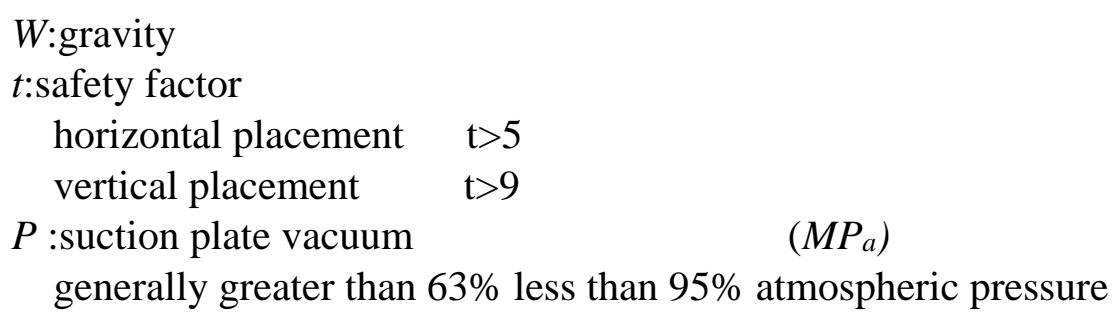

\section{Hardware Selection of Pneumatic System}

\section{The Electromagnetic Valve}

For the pneumatic system to work safely, to prevent sudden mechanical failure caused by mechanical parts, in the mechanical part of the interference with the cylinder we select the solenoid valve with self-holding function of the dual-pilot, for the other cylinder we selsect single-pilot electromagnetism valve:

Table 2. Electromagnetic valve selection

\begin{tabular}{c|c|c}
\hline Number & 1 & 2 \\
\hline Model & 4V220-08 & $4 \mathrm{~V} 210-08$ \\
\hline Specification & DC24V & DC24V \\
\hline Manufacturer & Airtic & Airtic \\
\hline Type & $\begin{array}{c}\text { Double electric control } \\
\text { pilot-operated }\end{array}$ & $\begin{array}{c}\text { Single electric control } \\
\text { pilot-operated }\end{array}$ \\
\hline
\end{tabular}

\section{The Air Cylinder}

In this system, the gas source working pressure is $0.6-0.8 \mathrm{MPa}$, according to the above section of the cylinder parameter determination method to determine the basic parameters of the cylinder, Part of the cylinder selection table:

Table 3. Air cylinder selection

\begin{tabular}{cccc}
\hline Number & Model & Specification & Manuf-acturer \\
\hline 1 & TN 32x60S & bore size $32 \mathrm{~mm}$ stroke $60 \mathrm{~mm}$ & Airtic \\
2 & TCL40x125S & bore size $40 \mathrm{~mm}$ stroke $125 \mathrm{~mm}$ & Airtic \\
3 & QGK-2SD25x180 & Output shaft diameter $25 \mathrm{~mm}$ & Airtic \\
4 & HFR20 & roating $180^{\circ}$ & Airtic \\
\hline Number & Type & Application workstation & \\
\hline 1 & double-shaft & bag-tidy & \\
2 & Tri-axial & bag-open & \\
3 & roating & bag-open & \\
4 & pneumatic gripper & bag-grasp & \\
\hline
\end{tabular}

Vacuum Chucks

There are many types of vacuum chucks, generally they are made by silica gel or nitrile butadiene 
rubber [5] .In this system, the sucker sucked items for the paper bag, in many tests on the basis of the final decision to use multi-lips type silicone vacuum suction cups. The use of a multi-lips-type structure makes the adsorption area becomes larger and improve the adsorption force.

Table 4. Vacuum chucks selection

\begin{tabular}{c|c|c}
\hline Model & Diameter & Material \\
\hline VB30S-18FB & $30 \mathrm{~mm}$ & silica gel \\
\hline Manufacturers & Connector & \\
\hline Vtec & G1/8 'nut & \\
\hline
\end{tabular}

\section{Experiment}

After the completion of all mechanical and control system design, the whole system has been debugged. The design can achieve all the scheduled functions. Cylinders and suction cups can perform predetermined movements such as extension, retraction, rotation, and suction. With the PLC control system, the device can complete manual and automatic control. The bagging speed up to 25 pices/min, so it meet the requirements.Figure 2 shows the finished product of this project.

\section{Summary}

The successful development of High-speed bagging machine solve the artificial bagging efficiency, labor-intensive issues. Which can automatically complete the supply and delivery of bags inside and outside the bag to take the bag, compound bag sewing process. In this paper, the theoretical analysis and calculation of the pneumatic system in the project are introduced, and the pneumatic components and the vacuum sucker are selected to complete the design of the pneumatic system. The program so that the lips and paper bag surface better fit, so as to prevent leakage occurs due to poor adsorption and enhance the adsorption capacity.

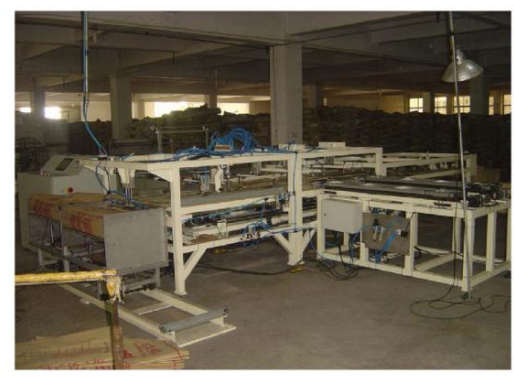

Figure 2. System on-site debugging

\section{References}

1. Wu Weirong. Pneumatic technologyM].China Light Industry Press, 2005: 2-4.

2. Frank M, White.Viscous Fluid F1ow.Mc Graw-Hill, 1974:589-592.

3. Xu Puzhu .Hydraulic and pneumatic, Pneumatic technology [M].Mechanical industry press, Beijing, 2005:19-24.

4. Zhang Pingping .The excogitation for the control system of the pneumatic manipulata (robot) based on PLC [D].School of Information and Software Engineering, 2013.

5. Li Xuemei, Zeng Dehuai, Ding Feng. The Design and Application of Vacuum cupule [J].Hydraulics \& Pneumatics, 2004, 03:48-49.

6. Andrikopoulos GSNikolakopoulos qManesis S. A survey on applications of pneumatic artificial 
muscles [C]. Control\&Automation (MED), 2011 19th Mediterranean Conference on. IEEE, 2011:1439-1446.

7. Villegas D, Van Damme M, Vanderburgh B, et a1.Third-Generation Pleated Pneumatic Artificial Muscles for Robotic Applications: Development and Comparison with McKibben Muscle [J].Advanced Robotics_2012_26(11-121: 1205-1227

8. K.W Gokhay. Design of PLC Control System. Electric Transmission, 2001, 33(7):13-15.

9. Xu Wencan.Selection of Components in Vacuum Circuits. [J] Hydraulics\&Pneumatics, 1998 (2):31-34.

10. John W Webb PronalA.Reis. Programmable Logic Controllers. Fourth Edition. Prentice Hall.Feb 1, 2006:30-36. 